\title{
ORTHOGONALITY PRESERVING MAPS AND THE LAGUERRE FUNCTIONAL
}

\author{
WILLIAM R. ALLAWAY
}

\begin{abstract}
Let $\Re[x]$ be the usual algebra of all polynomials in the indeterminate $x$ over the field of real numbers $\Re$, and let $\varphi$ be a linear operator mapping $\mathfrak{R}[x]$ into $\mathfrak{R}[x]$. In this paper we show that if $\varphi$ maps every orthogonal polynomial sequence into an orthogonal polynomial sequence, then $\varphi$ is defined by $\varphi\left(x^{n}\right)=s(a x+b)^{n}, n=0,1,2, \ldots$, where $s, a$, and $b$ belong to $\mathfrak{R}$, $s \neq 0$, and $a \neq 0$.
\end{abstract}

1. Introduction. Let $\mathfrak{R}[x]$ be the algebra of all polynomials in the indeterminate $x$ over the field of real numbers $\mathfrak{R} .\left\{p_{n}(x)\right\}_{n=0}^{\infty}$ is called a polynomial sequence if for all nonnegative integers $n, p_{n}(x)$ belongs to $\mathfrak{R}[x]$ and the degree of $p_{n}(x)$ equals $n$. Every polynomial sequence forms a basis of $\mathfrak{R}[x]$. A polynomial sequence $\left\{p_{n}(x)\right\}_{n=0}^{\infty}$ is called an orthogonal polynomial sequence (O.P.S.) with respect to the linear functional $L: \mathfrak{R}[x] \rightarrow \mathfrak{R}$, if there exist nonzero real constants $k_{n}$ such that for all nonnegative integers $n$ and $m$

$$
\mathbf{L}\left(p_{n}(x) p_{m}(x)\right)=k_{n} \delta_{n, m},
$$

where $\delta_{n, m}$ is the Kronecker delta. In (1.1), if $k_{n}=1$ for $n=0,1,2, \ldots$, then $\left\{p_{n}(x)\right\}_{n=0}^{\infty}$ is an orthonormal polynomial sequence. See Chihara $[\mathbf{3}]$ for an excellent treatise on orthogonal polynomial sequences.

Define the linear operator $\tau_{a, b}: \mathfrak{R}[x] \rightarrow \mathfrak{R}[x]$ by

$$
\tau_{a, b}\left(x^{n}\right)=(a x+b)^{n}, \quad n=0,1,2, \ldots,
$$

where $a$ and $b$ belong to $\mathfrak{R}$. If $s$ belongs to $R, a \neq 0, s \neq 0$, and $\left\{p_{n}(x)\right\}_{n=0}^{\infty}$ is an O.P.S. with respect to the linear functional $\mathbf{L}$, then $\left\{s \tau_{a, b}\left(p_{n}(x)\right)\right\}_{n=0}^{\infty}$ is an O.P.S. with respect to the linear functional $s^{-2} L \circ \tau_{1 / a,-b / a}$. This follows by noting that $\tau_{a, b}$ is an algebra automorphism on $\mathfrak{R}[x]$ and that $\tau_{1 / a,-b / a}$ is the inverse of $\tau_{a, b}$. This result is also true for orthonormal polynomial sequences.

We call a linear operator $\varphi: \mathfrak{R}[x] \rightarrow \mathfrak{R}[x]$ an orthogonality preserving map if it has the following properties:

(a) if $\left\{p_{n}(x)\right\}_{n=0}^{\infty}$ is an O.P.S., then $\left\{\varphi p_{n}(x)\right\}_{n=0}^{\infty}$ is an O.P.S.,

(b) $\varphi$ is degree preserving.

That is, if $\varphi$ is an orthogonality preserving map, then $\varphi$ maps every orthogonal polynomial sequence into an orthogonal polynomial sequence. For all real numbers $s, a$, and $b$, where $s, a \neq 0, s \tau_{a, b}$ is an example of an orthogonality preserving map.

Received by the editors January 31, 1986.

1980 Mathematics Subject Classification (1985 Revision). Primary 33A65.

Key words and phrases. Orthogonal polynomials, orthogonality preserving operators, Laguerre functional, Laguerre polynomials, shift operator, scale operator, pseudo-basis.

The author was supported in part by N.S.E.R.C. Grant A8721 and the President's N.S.E.R.C. Fund (Lakehead University). 
Al-Salam and Verma $\left[\mathbf{1}\right.$, Theorem 1] showed that the linear operator $J_{1}: \mathfrak{R}[x] \rightarrow$ $\mathfrak{R}[x]$ having the form

$$
J_{1} x^{n}=\sum_{k=0}^{n}\{n ! /((n-k) ! k !)\} b_{n-k} x^{k}, \quad n=0,1,2, \ldots,
$$

is an orthogonality preserving map if and only if $J_{1}=s \tau_{1, b}$. They also remarked in [2] that the linear operator $J_{2}$ having the form $J_{2} x^{n}=j_{n} x^{n}, n=0,1,2, \ldots$, is an orthogonality preserving map if and only if $J_{2}=s \tau_{a, 0}$. This paper is a generalization of the work of Al-Salam and Verma $[\mathbf{1}, 2]$ in that our main result is the following theorem.

THEOREM (1.1). If $\varphi$ is an orthogonality preserving map, then there exist $s$, $a$, and $b$ all belonging to $\mathfrak{R}$ such that $\varphi=s \tau_{a, b}$.

This theorem is proved by proving a stronger result; namely, if $\varphi$ maps certain Laguerre polynomial sequences into orthogonal polynomial sequences, then $\varphi=$ $s \tau_{a, b}$ (see Theorem (4.1)).

2. A pseudo-basis for the dual of $\mathfrak{R}[x]$. Let $\alpha$ be a real number, not equal to a negative integer. It is well known [3] that the Laguerre polynomial sequence $\left\{L_{n}^{\alpha}(x)\right\}_{n=0}^{\infty}$ is orthogonal with respect to the linear functional $\mathrm{F}_{\alpha}: \mathfrak{R}[x] \rightarrow \mathfrak{R}$ defined by

$$
\mathrm{F}_{\alpha}\left(x^{i}\right)=\int_{0}^{\infty} x^{\alpha} \exp (-x) x^{i} d x / \Gamma(\alpha+1),
$$

where $\Gamma(\alpha+1)$ is the gamma function. The orthonormal Laguerre polynomial sequence $\left\{\mathbf{L}_{n}^{\alpha}(x)\right\}_{n=0}^{\infty}$ can be defined by $\mathbf{L}_{n}^{\alpha}(x)=\left(n ! /(1+\alpha)_{n}\right)^{1 / 2} L_{n}^{\alpha}(x)$, where $(1+\alpha)_{n}=(1+\alpha)(2+\alpha) \cdots(n+\alpha)$.

We define the Laguerre functional $\mathfrak{S}_{n}^{\alpha}: \mathfrak{R}[x] \rightarrow \mathfrak{R}$ by

$$
\mathbb{S}_{n}^{\alpha}\left(x^{i}\right)=\int_{0}^{\infty} \mathbf{L}_{n}^{\alpha}(x) x^{\alpha} \exp (-x) x^{i} d x / \Gamma(\alpha+1)
$$

for $n, i=0,1,2, \ldots$ Thus, for $n, m=0,1,2,3, \ldots$,

$$
\mathrm{S}_{n}^{\alpha}=\sqrt{n !(1+\alpha)_{n}} \sum_{k=0}^{n}\left\{(-1)^{k} /((n-k) ! k !)\right\} \mathrm{F}_{\alpha+k}
$$

and

$$
\mathbb{S}_{n}^{\alpha}\left(\mathbf{L}_{m}^{\alpha}(x)\right)=\delta_{n, m}
$$

Roman and Rota [4] call $\left\{S_{n}^{\alpha}\right\}_{n=0}^{\infty}$ a pseudo-basis of the dual of $\Re[x]$. That is, every linear functional $\mathrm{M}$ can be written in the form

$$
\mathbf{M}=\sum_{k=0}^{\infty} m_{k} \mathbb{S}_{k}^{\alpha}
$$

where $m_{k}=\mathbf{M}\left(\mathbf{L}_{k}^{\alpha}(x)\right)$. If the degree of the polynomial $q(x)$ is $n$, then for all $k>n$, $\mathrm{S}_{k}^{\alpha}(q(x))=0$. Thus, when the infinite sum of linear functionals on the right-hand side of (2.4) acts on $q(x)$, the infinite sum collapses into a finite sum of at most $n+1$ terms.

The next proposition is a major tool used in proving Theorem (1.1). 
Proposition (2.1). Let $\varphi: \mathfrak{R}[x] \rightarrow \mathfrak{R}[x]$ be a fixed, degree preserving linear operator, such that $\varphi(1)=s$ and $\varphi(x)=s(a x+b)$. If there exists $\alpha$, not a negative integer, such that for $i, n=0,1,2, \ldots$,

$$
s \mathrm{~F}_{\alpha+i}\left(x L_{n}^{\alpha+i}(x)\right)=\mathrm{F}_{\alpha+i}\left(\varphi^{-1}\left((\varphi x)\left(\varphi L^{\alpha+i}(x)\right)\right)\right),
$$

then $\varphi=s \tau_{a, b}$.

ProOF. By using (2.2), (2.5), and the fact that $\left\{L_{n}^{\alpha}(x)\right\}_{n=0}^{\infty}$ forms a basis of $\mathfrak{R}[x]$, it is easy to show that for all polynomials $q(x)$

$$
s \aleph_{n}^{\alpha}(x q(x))=\$_{n}^{\alpha}\left(\varphi^{-1}((\varphi x)(\varphi q(x)))\right),
$$

where $n=0,1,2,3, \ldots$. Define the evaluation functional at $a, \mathrm{E}_{a}$, by $\mathrm{E}_{a}(q(x))=$ $q\left(a^{\prime}\right)$. Since $\left\{\mathbb{S}_{n}^{\alpha}\right\}_{n=0}^{\infty}$ is a pseudo-basis of the algebraic dual of $\mathfrak{R}[x], \mathrm{E}_{a}$ can be written in the form

$$
\mathrm{E}_{a}=\sum_{k=0}^{\infty} \mathbf{L}_{k}^{\alpha}(a) \mathbb{S}_{k}^{\alpha} .
$$

Thus, for all real numbers $a$ and all polynomials $q(x)$.

$$
s \mathrm{E}_{a}(x q(x))=\mathrm{E}_{a}\left(\varphi^{-1}((\varphi x)(\varphi q(x)))\right),
$$

which implies that $s \varphi\left(x x^{n}\right)=\varphi(x) \varphi\left(x^{n}\right)$ for $n=0,1,2, \ldots$ By a simple induction argument we obtain $\varphi=s \tau_{a, b}$. Q.E.D.

\section{The orthonormal case.}

THEOREM (3.1). If there exists a real number $\alpha$, which is not a negative integer, such that $\left\{\varphi \mathbf{L}_{n}^{\alpha+i}(x)\right\}_{n=0}^{\infty}$ is an orthonormal polynomial sequence for $i=$ $0,1,2, \ldots$, then there exist real numbers $s, a$, and $b$, where $s, a \neq 0$, such that $\varphi=s \tau_{a, b}$.

PROOF. There exist real numbers $s, a$, and $b$, where $s, a \neq 0$, such that $\varphi(1)=s$, $\varphi(x)=s(a x+b)$. Let $\mathbf{M}_{\alpha, i}$ be the linear functional for which $\left\{\varphi \mathbf{L}_{n}^{\alpha+i}(x)\right\}_{n=0}^{\infty}$ is orthonormal. Thus for $i, n=0,1,2, \ldots$,

$$
s \mathbf{M}_{\alpha, i} \circ \varphi\left(\mathbf{L}_{n}^{\alpha+i}(x)\right)=\delta_{n, 0}=\mathrm{F}_{\alpha+i}\left(\mathbf{L}_{n}^{\alpha+i}(x)\right)
$$

and

$$
\mathbf{M}_{\alpha, i}\left(\varphi\left(\mathbf{L}_{1}^{\alpha+i}(x)\right) \varphi\left(\mathbf{L}_{n}^{\alpha+i}(x)\right)\right)=\delta_{n, 1}=\mathrm{F}_{\alpha+i}\left(\mathbf{L}_{1}^{\alpha+i}(x) \mathbf{L}_{n}^{\alpha+i}(x)\right) .
$$

It is easy to show that (3.1) and (3.2) imply (2.5). Thus by Proposition (2.1) we have $\varphi=s \tau_{a, b}$. Q.E.D.

4. The orthogonal case. To prove the orthogonal case we need a hypothesis that is slightly stronger than the hypothesis in Theorem (3.1).

THEOREM (4.1). Let $\varphi$ be a fixed linear operator. If there exists $\alpha_{1}<-1$, not a negative integer, and $\alpha_{2}>-1$ such that, for $\alpha=\alpha_{i}+j, i=1,2, j=$ $0,1,2, \ldots,\left\{\varphi L_{n}^{\alpha}((x-d) / c)\right\}_{n=0}^{\infty}$ is an orthogonal polynomial sequence for $c$ any nonzero real number and $d$ any real number, then there exist real numbers $s, a$, and $b$ (independent of $\alpha_{1}$ and $\alpha_{2}$ ) such that $\varphi=s \tau_{a, b}$.

PROOF. Because $\varphi$ is degree preserving, therefore there exist real numbers $s, a$, $b, t_{2,2}, t_{2,1}$, and $t_{2,0}$ such that $s, a, t_{2,2} \neq 0$,

$$
\varphi\left(x^{i}\right)=s(a x+b)^{i} \quad \text { for } i=0 \text { and } 1,
$$


and

$$
\varphi\left(x^{2}\right)=s\left(t_{2,2} x^{2}+t_{2,1} x+t_{2,0}\right) .
$$

By using a method similar to that used in the above orthonormal case, we have for $\alpha=\alpha_{1}+j, i=1,2, j=0,1,2, \ldots$;

$$
s \mathrm{~F}_{\alpha}\left(x L_{n}^{\alpha}(x)\right)=\mathrm{F}_{\alpha} \circ \varphi^{-1}\left((\varphi x)\left(\varphi L_{n}^{\alpha}(x)\right)\right),
$$

where $n$ is any nonnegative integer not equal to 1. Because of Proposition (2.1), in order to obtain the conclusion of the theorem, we need only show that (4.3) is also true for $n=1$. This is equivalent to showing that $\varphi\left(x^{2}\right)=s(a x+b)^{2}$.

Let $m_{0}, m_{1}, m_{2}$ be any three real numbers such that $m_{0} \neq 0$ and

$$
m_{0} m_{2}-m_{1}^{2}=0 .
$$

Define the real numbers $\mu_{0}, \mu_{1}$, and $\mu_{2}$ in terms of $m_{0}, m_{1}$, and $m_{2}$ by

$$
\left\{\begin{array}{l}
\mu_{0}=s m_{0} \\
\mu_{1}=s\left(a m_{1}+b m_{0}\right) \\
\mu_{2}=s\left(t_{2,2} m_{2}+t_{2,1} m_{1}+t_{2,0} m_{0}\right)
\end{array}\right.
$$

where $s, a, b, t_{2,2}, t_{2,1}$, and $t_{2,0}$ are defined in terms of $\varphi$ by (4.1) and (4.2).

We wish to show that $\mu_{0} \mu_{2}-\mu_{1}^{2}=0$.

Assume that $\mu_{0} \mu_{2}-\mu_{1}^{2} \neq 0$. From this statement, it is easy to show the existence of a real number $\mathbf{d}$ such that $\left(\mu_{1}-\mathbf{d} \mu_{0}\right)^{2}\left(\mu_{0} \mu_{2}-\mu_{1}^{2}\right)^{-1}-1=\alpha$, where $\boldsymbol{\alpha}=\alpha_{1}$ or $\alpha_{2}$ and for $n=0,1$ and 2

$$
\mu_{0} \mathrm{~F}_{\boldsymbol{\alpha}}\left(\tau_{\mathbf{c}, \mathbf{d}}\left(x^{n}\right)\right)=\mu_{n},
$$

where $\mathbf{c}=\left(\mu_{2} \mu_{0}-\mu_{1}^{2}\right)\left(\mu_{0}\left(\mu_{1}-\mu_{0} \mathbf{d}\right)\right)^{-1}$. By hypothesis $\left\{\varphi L_{n}^{\alpha}((x-\mathbf{d}) / \mathbf{c})\right\}_{n=0}^{\infty}$ is an O.P.S. with respect to a quasi-definite linear functional which we will denote by $\mathbf{M}_{\boldsymbol{\alpha}, \mathbf{d}, \mathbf{c}} \cdot \mathbf{M}_{\boldsymbol{\alpha}, \mathbf{d}, \mathbf{c}}$ can be chosen in such a way that

$$
\mu_{0} \mathrm{~F}_{\boldsymbol{\alpha}}\left(\tau_{\mathbf{c}, \mathbf{d}}\left(L_{n}^{\boldsymbol{\alpha}}((x-\mathbf{d}) / \mathbf{c})\right)\right)=\delta_{n, 0} \mu_{0}=\mathbf{M}_{\boldsymbol{\alpha}, \mathbf{d}, \mathbf{c}}\left(\varphi\left(L_{n}^{\boldsymbol{\alpha}}((x-\mathbf{d}) / \mathbf{c})\right)\right) .
$$

But $\left\{\varphi L_{n}^{\alpha}((x-\mathbf{d}) / \mathbf{c})\right\}_{n=0}^{\infty}$ forms a basis of $\Re[x]$, thus we have the functional equality

$$
\mu_{0} F_{\alpha} \circ \tau_{\mathbf{c}, \mathbf{d}}=\mathbf{M}_{\boldsymbol{\alpha}, \mathbf{d}, \mathbf{c}} \circ \varphi
$$

By using (4.5), (4.6), and (4.7), we have that $\mathbf{M}_{\alpha, \mathrm{d}, \mathrm{c}}\left(x^{i}\right)=m_{i}, i=0,1$ and 2 . But $\mathbf{M}_{\boldsymbol{\alpha}, \mathbf{d}, \mathbf{c}}$ is a quasi-definite linear functional and therefore $m_{0} m_{2}-m_{1}^{2} \neq 0$, which contradicts (4.4). Thus our assumption that $\mu_{0} \mu_{2}-\mu_{1}^{2} \neq 0$ is incorrect and therefore

$$
\left|\begin{array}{ll}
\mu_{0} & \mu_{1} \\
\mu_{1} & \mu_{2}
\end{array}\right|=\left|\begin{array}{ll}
s m_{0} & s\left(a m_{1}+b m_{0}\right) \\
s\left(a m_{1}+b m_{0}\right) & s\left(t_{2,2} m_{2}+t_{2,1} m_{1}+t_{2,0} m_{0}\right)
\end{array}\right|=0 .
$$

That is, for all nonzero real numbers $m_{0}$ and $m_{1}$,

$$
\left(t_{2,2}-a^{2}\right) m_{1}^{2}+\left(t_{2,1}-2 a b\right) m_{0} m_{1}+\left(t_{2,0}-b^{2}\right) m_{0}^{2}=0 .
$$

Thus $\varphi\left(x^{i}\right)=s(a x+b)^{i}$ for $i=0,1$ and 2. Q.E.D.

Theorem (4.1) implies Theorem (1.1). 
5. A conjecture. Because of Theorems (3.1) and (4.1), it seems reasonable to conjecture that, if there exists a real number $\alpha$, which is not a negative integer such that $\left\{\varphi L_{n}^{\alpha+i}(x)\right\}_{n=0}^{\infty}$ is an orthogonal polynomial sequence for $i=0,1,2,3, \ldots$, then there exist real numbers $s, a$, and $b$, where $s, a \neq 0$, such that $\varphi=s \tau_{a, b}$. Such a result would be stronger than Theorem (4.1).

6. Acknowledgment. We would like to express our sincere thanks to Professor Bruce Char and Mr. Greg Fee of the Maple Group at the University of Waterloo for solving a system of 21 nonlinear equations in 18 unknowns symbolically on their Honeywell Computer. Their solution not only showed that the conjecture given in $\S 5$ is most likely true but it also showed that the technique we were using to try and prove it will not succeed.

\section{REFERENCES}

1. W. A. Al-Salam and A. Verma, Some orthogonality preserving operators, Proc. Amer. Math. Soc. 23 (1969), 136-139.

2. __ Orthogonality preserving operators, Accad. Naz. Lincei 58 (1975), 833-838.

3. T. S. Chihara, An introduction to orthogonal polynomials, Gordon and Breach, New York, 1978.

4. S. Roman and G.-C. Rota, The umbral calculus, Adv. in Math. 27 (1978), 95-188.

DEPARTMENT OF MATHEMATICAL SCIENCES, LAKEHEAD UNIVERSITy, THUNDER BAy, ONTARIO, CANADA P7B 5E1 